\title{
Hybrid Packet/Fluid Flow Network Simulation
}

\author{
Cameron Kiddle, Rob Simmonds, Carey Williamson, and Brian Unger \\ \{kiddlec,simmonds,carey,unger\}@ cpsc.ucalgary.ca \\ Department of Computer Science \\ University of Calgary \\ Calgary, Alberta, Canada
}

\begin{abstract}
Packet-level discrete-event network simulators use an event to model the movement of each packet in the network. This results in accurate models, but requires that many events are executed to simulate large, high bandwidth networks. Fluid-based network simulators abstract the model to consider only changes in rates of traffic flows. This can result in large performance advantages, though information about the individual packets is lost making this approach inappropriate for many simulation and emulation studies.

This paper presents a hybrid model in which packet flows and fluid flows coexist and interact. This enables studies to be performed with background traffic modeled using fluid flows and foreground traffic modeled at the packet level. Results presented show up to 20 times speedup using this technique. Accuracy is within 4\% for latency and $15 \%$ for jitter in many cases.
\end{abstract}

Keywords: Network Simulation, Simulation Abstraction Techniques, Fluid Simulation, Scalable Network Simulation

\section{Introduction}

Discrete-event network simulators often model traffic at the packet level, with an event being used to represent packet arrivals or departures from network devices or buffers. This can lead to accurate models. However, when simulating large networks and high bandwidth links, the computational cost of processing the resulting huge number of events representing the traffic as packet flows can be prohibitive. When simulators are used within real-time network emulation environments, this cost severely restricts the size and type of network that can be modeled.

Parallel discrete-event simulation (PDES) techniques can increase model scalability, i.e., the size of network and the traffic densities that can be executed in real-time. Modeling larger bandwidth links is less amenable to parallelization techniques due to the sequential nature of each packet flow at each network port. Therefore, model abstraction techniques are required to simulate large traffic flows.

Fluid-based modeling can be used to simplify traffic flows in a network simulation $[2,3,4,8,9]$. With a fluid model, events are only generated when the rate of a flow changes. If the flows change rate infrequently, large performance gains can be achieved using this technique. Since model detail is reduced, the level of accuracy of the simulation results obtained using this abstraction technique will not be as high as when packet-level simulation is used. As with all abstraction techniques, the appropriateness of the method depends on the simulation requirements.

One problem with fluid models is that information about individual packets is lost. Therefore, they cannot be used for simulations studying subtle protocol dynamics on individual flows. They can also not be used for simulators that act as components of network emulation systems that interact with real applications running on real networks. These real applications communicate using individual packets, so a simulator interacting with them must handle individual packets.

One approach to maintaining packet information while reducing the overall traffic modeling cost is to use hybrid simulators that handle both packet and fluid flows $[5,11$, 13]. Traffic flows that must carry the full packet information are modeled using an event for each packet arrival or departure while background flows, for which less detailed information is required, are modeled using fluid flows. A challenge faced by these systems is accurately modeling the interactions between packet flows and fluid flows.

This paper describes the design of a hybrid model that has been implemented within a parallel IP packet-level network simulator called the Internet Protocol Traffic and Network (IP-TN) simulator [12]. This simulator forms the basis of the IP-TNE network emulation system making it essential that abstraction techniques employed do not prohibit the modeling of individual packets. Results are presented showing the performance and accuracy achieved.

The rest of the paper is laid out as follows. Section 2 describes related work in the area. Section 3 describes the de- 
sign of the hybrid model. Section 4 presents the experimental methodology and Sections 5 and 6 present the results. Conclusions and future work are presented in Section 7.

\section{Related Work}

The accuracy and/or performance of fluid-based techniques for network simulation have been examined in [2, $3,4,8,9]$. Reasonable accuracy has been achieved along with considerable performance improvement under certain circumstances. Compared to packet-level simulation, the largest performance gains are achieved with small networks and cases where the number of packets represented is much larger than the number of rate changes. For larger networks, a property described as the ripple effect can reduce the performance advantage in the fluid-based simulator. The ripple effect describes the situation where the propagation of rate changes leads to rate changes in other flows which then need to be propagated.

On/Off sources are commonly used as traffic models in the fluid simulation literature. The use of these models makes it easy to study accuracy and performance issues. Some work has involved the use of fluid-based TCP models $[6,7]$.

The hybrid technique in which packet flows and fluid flows are integrated is a recent development. An extension adding fluid models to the QualNet simulator is presented in [13]. QualNet is the commercial version of Global Mobile Information System Simulator (GloMoSim) [14]. The simulated system is divided into components that model at the packet level and components that use analytical fluidbased models. The fluid-based components calculate delays and loss of traversing packet flows and pass this information to the destination packet modeling components.

The Hybrid Discrete-Continuous Flow Network Simulator (HDCF-NS) [5] also enables packet flows and fluid flows to coexist. The manner in which fluid flows are modeled is described in detail, but little information is given on how the packet and fluid flows interact.

A third hybrid approach is described in [11]. This utilizes two simulators that interact via the Georgia Tech Runtime Infrastructure Kit (RTIKIT) [1]. The HDCF-NS simulator is used to simulate the background fluid flows and the parallel and distributed ns (pdns) simulator [10] is used to simulate the packet flows. Messages are sent to pdns from HDCF-NS via RTIKIT whenever fluid changes occur. Packet loss and queuing delay in pdns are based on both packet and fluid levels. The system currently does not allow packet flows to affect fluid flows.

\section{Hybrid Implementation}

The hybrid scheme presented in this paper is similar to the one used in HDCF-NS since it combines packet and fluid discrete-event models in a single simulator. However, little information is given in [5] on the hybrid implementation details of HDCF-NS. QualNet differs from our approach in that different sections of the network support either fluid or packet flows but not both. Also the fluid sections use an analytical model instead of a discrete-event model. The approach using both HDCF-NS and pdns differs from the approach taken here in that multiple simulators are used and packet flows cannot affect background fluid flows.

The approach taken in this paper adds fluid modeling to the IP-TN simulator by adding a new type of output buffer at network links. This hybrid buffer must be able to process packets and fluid advertisements. A fluid advertisement specifies a rate change for a particular fluid flow. It is possible for a packet and multiple advertisements to be sent at the same time; in this case they are represented by a single event. The IP routing lookup was modified to demultiplex packets and advertisements that arrive together.

The hybrid buffer models a FIFO queue that operates in one of three modes: packet mode, fluid mode or hybrid mode. Initially, the mode of the buffer is undefined. If a buffer first receives a packet, it enters packet mode and remains in packet mode as long as it only processes packets. If a buffer first receives a fluid advertisement, it enters fluid mode and remains in fluid mode as long as it only processes fluid advertisements. A buffer switches to hybrid mode if it is in packet mode and receives a fluid advertisement or if it is in fluid mode and receives a packet. Currently, once a buffer switches into hybrid mode, it remains in hybrid mode for the remainder of the simulation. The hybrid buffer modes are described in the following sections.

\subsection{Packet Mode}

Operation of the hybrid buffer in packet mode is depicted in Figure 1, where $x(t)$ is the buffer usage at time $t, x_{C}$ is the maximum buffer capacity and $\lambda$ is the buffer service rate, which is the bandwidth of the outgoing link. If there is

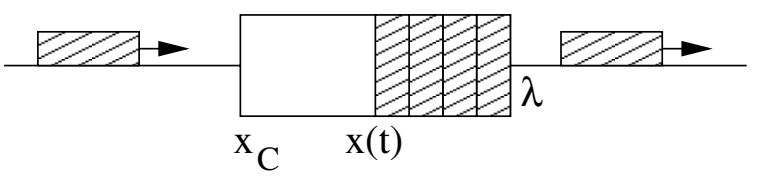

Figure 1. Hybrid buffer in packet mode.

insufficient space in the buffer, the packet is dropped when it arrives. Otherwise, the packet is added to the buffer and the arrival time at the next node calculated based on queuing delay, transmission delay and propagation delay. A new event to represent the packet is generated and dispatched to the next node in the network. 


\subsection{Fluid Mode}

In fluid mode, state is maintained for each fluid flow indicating its advertised incoming rate $\alpha_{i}(t)$, advertised outgoing rate $\beta_{i}(t)$ and loss rate $l_{i}(t)$ at time $t$. Other state maintained by the buffer includes the aggregate input rate $\alpha(t)$, the aggregate output rate $\beta(t)$, the aggregate loss rate $l(t)$, the buffer usage $x(t)$, the buffer capacity $x_{C}$ and the buffer service rate $\lambda$, which is the bandwidth of the outgoing link. Operation of the buffer in fluid mode is depicted in Figure 2.

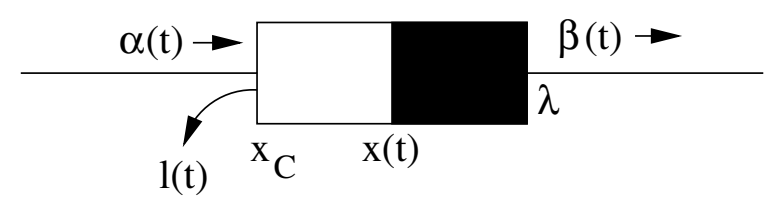

Figure 2. Hybrid buffer in fluid mode.

When a fluid advertisement(s) arrives at time $t$, the buffer usage, packet loss, the new aggregate input rate and output flow rates are calculated. The buffer usage $x(t)$ is calculated as follows:

$$
x(t)=x\left(t_{l}\right)+\left(\alpha\left(t_{l}\right)-\lambda\right) *\left(t-t_{l}\right),
$$

where $t_{l}$ is the time at which the last fluid advertisements arrived. If the calculated value of $x(t)<0$ then $x(t)$ is set to be 0 . If the calculated value of $x(t)>x_{C}$ then $x(t)$ is set to be $x_{C}$. Loss rates and output rates of individual flows at time $t$ are calculated after $x(t)$ has been determined as follows:

1. (Underload/Empty) if $\alpha(t)<\lambda$ and $x(t)=0$ then $\beta_{i}(t)=\alpha_{i}(t)$ and $l(t)=0$.

2. (Underload/Draining) if $\alpha(t)<\lambda$ and $0<x(t) \leq x_{C}$ then $\beta_{i}(t)=\frac{\alpha_{i}(t)}{\alpha(t)} \times \lambda$ and $l(t)=0$. (Note that an event must be generated for the predicted time that the buffer will empty, so output rates can be re-advertised.)

3. (Overload/Filling) if $\alpha(t) \geq \lambda$ and $0 \leq x(t)<x_{C}$ then $\beta_{i}(t)=\frac{\alpha_{i}(t)}{\alpha(t)} \times \lambda$ and $l(t)=0$. (Note that an event does not need to be generated for the predicted time that the buffer will become full, as the output rates of the flows will remain the same. Packet loss can be determined upon arrival of the next set of fluid advertisements.)

4. (Overload/Loss) if $\alpha(t) \geq \lambda$ and $x(t)=x_{C}$ then $\beta_{i}(t)=\frac{\alpha_{i}(t)}{\alpha(t)} \times \lambda, l(t)=\alpha(t)-\lambda$ and $l_{i}(t)=$ $\frac{\alpha_{i}(t)}{\alpha(t)} \times l(t)$.

For each flow in which the output rate changed, a fluid advertisement is created. The next hop arrival time of the fluid advertisements is calculated based on queuing and the propagation delay of the link. An event is then generated to signal the arrival of the fluid advertisements at the next hop at the time calculated. Note that transmission delay is not included in the calculation of the arrival time. Characterizing the transmission delay is difficult since different flows may have different packet sizes. Usually, propagation delay is significantly larger than transmission delay and therefore should not introduce much inaccuracy.

\subsection{Hybrid Mode}

In hybrid mode, a single buffer that operates in a similar way as the buffer in fluid mode is used. As both packet flows and fluid flows are handled, it is important that packet flow levels affect fluid flow levels and vice versa. This is achieved by estimating the aggregate input rate of packet flows $\alpha_{p}(t)$ and adding this to the aggregate input rate of fluid flows $\alpha_{f}(t)$ to get the overall aggregate input rate $\alpha(t)$. An output rate is not advertised for the packet flows so there is only an aggregate output rate of fluid flows $\beta_{f}(t)$. The operation of the buffer in hybrid mode can be seen in Figure 3 .

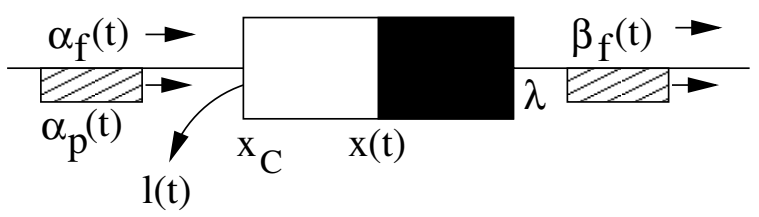

Figure 3. Hybrid buffer in hybrid mode.

The process and calculations involved for handling fluid advertisements is the same as for fluid mode except that the current value for $\alpha_{p}(t)$ is included as part of $\alpha(t)$. Fluid advertisements are sent for all fluid flows with rate changes.

Each time a packet arrives, a new $\alpha_{p}$ estimate is calculated. The estimate is based on a sliding window of the last $X$ packets that have arrived. It is calculated by taking the sum of the size of the last $X$ packets and dividing it by the time interval in which the last $X$ packets arrived. Other rate estimation algorithms based on exponentially weighted moving averages and time windows were explored but were found to be too sensitive to parameter settings.

Once the new $\alpha_{p}$ estimate has been calculated it is compared with the current $\alpha_{p}$. If the difference between the new estimate and the current rate is greater than a threshold percentage, then the current rate is set to the new estimate. Modifying $\alpha_{p}$ each time a packet arrives could lead to a greater impact from the ripple effect in fluid flows. The threshold value allows for some control in balancing accuracy and performance.

Each time $\alpha_{p}$ is modified, a timeout event is generated. This allows $\alpha_{p}$ to be reset to zero during a period when no packets are arriving. The timeout is set to occur when it is 
expected that $Y$ packets will have arrived according to the current $\alpha_{p}$. When the timeout occurs, $\alpha_{p}$ is set to zero if no packets arrived since the timeout was generated. Otherwise, a new timeout is generated in the same manner as before. The next time a packet arrives, the last $X$ packets are still used in the $\alpha_{p}$ estimation.

For each packet arrival, buffer usage is calculated as in fluid mode. Loss in fluid flows, the aggregate input rate and new output rates of fluid flows are calculated if $\alpha_{p}$ was modified, or if the packet arrived with fluid advertisements. If the buffer is full, the packet is lost with probability $\frac{(\alpha-\lambda)}{\alpha}$, which is the same proportion in which fluid flows lose packets. If the packet is not lost, it is sent along with any fluid advertisements that may have been created due to a change in $\alpha_{p}$. The arrival time at the next network node is calculated based on the queuing delay and propagation delay as is done in fluid mode.

Since fluid modeling principles are used, the transmission delay of a packet is ignored. Also, if two packets were to arrive at the buffer at the same time, they would both arrive at the next network node at the same time. To account for transmission delays and queuing among packet flows, an alternative solution with two buffers could be used. One buffer could handle packets and the other buffer could handle fluid flows. Effects of fluid flows on packet flows could be modeled by adjusting buffer usage of the packet buffer based on the amount of data arriving from fluid flows. An estimate of the aggregate input rate of packet flows could be used to affect the output rates and loss of the fluid flows.

The alternative solution could potentially lead to more accurate results, but would be difficult to implement efficiently. Buffer usage in the two buffers could differ and this could result in events that signal arrivals of packets and fluid advertisements being sent out of arrival time order. IP-TN uses conservative parallel discrete-event simulation techniques, which require that events sent between nodes are received in arrival time order. To achieve this, the alternative solution would require two events, instead of just one event, to be generated each time a packet or set of fluid advertisements is to be sent. One event would be used to signal the actual sending of the packet or set of fluid advertisements. The other event would be used to signal the arrival of the packet or set of fluid advertisements at the next hop. The send events would be processed in send time order ensuring that arrival events are received in arrival time order.

\section{Experimental Methodology}

This section describes the methodology for the simulation experiments, including the experimental environment, network model, experimental design, and performance metrics.

\subsection{Experimental Environment}

Simulation experiments were conducted on an 8processor Compaq Proliant server with $700 \mathrm{MHz}$ Intel PIII Xeon processors and 4 GB RAM. The Proliant was running RedHat Linux 7.3 with the v2.4.18 kernel. The GNU $\mathrm{g}++\mathrm{V} 2.96$ compiler was used with the -O2 optimization flag. Tests were run using sequential execution on one processor to examine effects of just the hybrid model without interference from the effects of parallelism. In runs comparing packet and hybrid simulations, identical random number seeds were used.

\subsection{Network Model}

The network model used for the simulation experiments is shown in Figure 4. This model is a variation of the classic "tandem" network model, but with bi-directional links. With this model, we can test the performance and the accuracy of our hybrid simulation methodology as the size of the network and the number of traffic flows are varied.

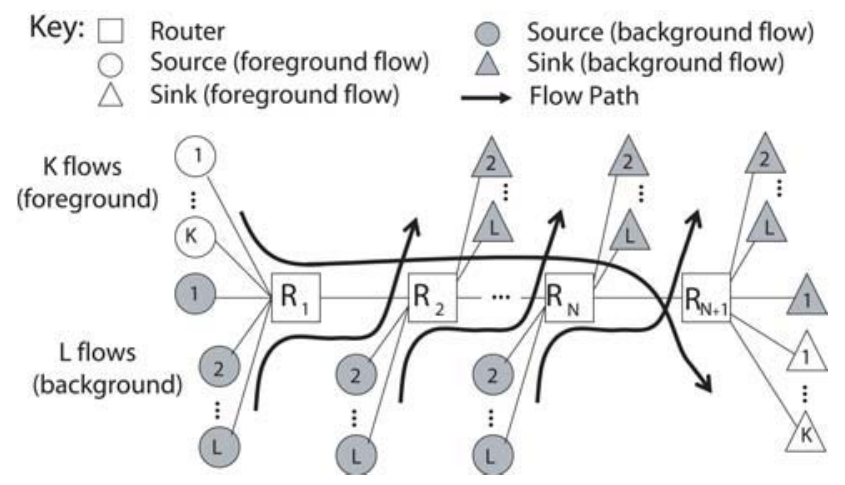

Figure 4. Simulated network model.

Three main parameters characterize the network model: $K, L$, and $N . K$ is the number of foreground flows in the network. These flows traverse the entire backbone of the network, from end to end. $L$ is the number of background flows on each link in the network backbone. One of the background flows traverses the entire network. The other background flows each traverse only a single link in the network backbone. The total number of traffic flows on any given backbone link is $M=K+L$. These $M$ flows compete for resources on the network (i.e., for network bandwidth, and for the buffers at the router output queues). $N$ is the number of links (router hops) in the backbone of the network. In particular, $N$ is the number of queues at which flows interact and compete for resources.

The details of the network topology model are as follows. Links from the traffic sources to routers have a 1 millisecond propagation delay and $10 \mathrm{Mbps}$ transmission capacity, as do the links from routers to the traffic sinks. Each 
link in the network backbone has a 5 millisecond propagation delay and transmission capacity $C \mathrm{Mbps}$. This capacity is set to achieve different target levels of offered network load in the experiments. Output buffers on routers have a maximum size that corresponds to 20 milliseconds of queuing delay for a link capacity of $C$ Mbps.

In our experiments, the foreground flows are always modeled as packet flows. The background flows are modeled either as fluid flows or as packet flows, depending on the simulation experiment.

\subsection{Traffic Source Models}

Two different types of traffic source models are used in the experiments: open-loop and closed-loop. An open-loop model generates traffic according to its statistical parameters, independent of the state of the network. That is, there is no feedback control in the model. All traffic moves unidirectionally from source to sink in our network model. A closed-loop model has a built-in feedback loop for traffic control. Data packets flow from source to sink in our network model, while acknowledgment packets traverse the reverse route.

The open-loop source model used is an Exponential On/Off traffic source. In the On state, the source generates traffic at a specified peak rate of $5 \mathrm{Mbps}$. In the Off state, no traffic is generated. The sojourn times in each state are drawn independently from an exponential distribution with a specified mean. In our model, each source spends (on average) $50 \%$ of the time in each state with a resulting mean rate of $2.5 \mathrm{Mbps}$. During each On period, an average of 100 packets of size 576 bytes are generated. Both packet-based and fluid-based versions of this model exist in IP-TN.

The closed-loop source model used is a simulated version of TCP Reno. This protocol model includes TCP's three-way handshake, sequence numbers, acknowledgments, sliding window flow control, slow-start, congestion avoidance, timeouts, and retransmissions. In particular, each TCP source transmits packets according to TCP's flow control and congestion control algorithms. Only a packetbased version of this model exists in IP-TN at this time.

\subsection{Experimental Design}

Table 1 summarizes the experimental factors and levels used in the experiments. A multi-factor experimental design is used. For space reasons, only a subset of these experiments appear in the paper. Packet rate estimation algorithm parameter values of $X=10, Y=10$ and threshold $=1 \%$ were used.

\subsection{Performance Metrics}

The performance metrics fall into two main categories: metrics for simulation execution-time performance, and
Table 1. Experimental factors and levels for simulation experiments.

\begin{tabular}{|l|l|}
\hline Factor & Levels \\
\hline Foreground Flows $K$ & $1,2,4$ \\
Background Flows $L$ & $2,4,8,16,32$ \\
Router Hops $N$ & $1,2,4,8,16$ \\
\hline Traffic Flow Type & Open-loop, Closed-loop \\
Background Flow Model & Packet, Fluid \\
Network Load & Light(25\%), Medium(70\%), \\
& Heavy(100\%) \\
\hline
\end{tabular}

metrics for quantifying the results of the network simulation. The latter network-centric metrics are used to assess the accuracy of the hybrid simulation results compared to the packet simulation results.

The metric used for simulation execution-time performance is relative speedup. This is defined as the ratio of the execution time for the packet simulation to the execution time for the hybrid simulation. Higher values of this metric indicate performance advantages for the hybrid simulation.

The metrics used for assessing network-level performance include the mean end-to-end packet transfer latency and the jitter (e.g., standard deviation) of the end-to-end packet transfer latency. For simplicity, these performance metrics are calculated for only one of the foreground traffic flows in the network, called the primary flow. The results then focus on the relative error in these metrics for the primary flow in the hybrid simulation. That is, we express the latency results from the hybrid simulation as a percentage difference from the latency results for the packet simulation. A similar calculation applies for the jitter metric.

The experiments with the closed-loop TCP traffic model use one additional metric, namely the TCP transfer duration. This metric represents the elapsed time between receiving the first byte and the last byte of a TCP data transfer. This metric is used to assess the cumulative modeling error over the duration of a multi-packet TCP transfer.

\section{Results for Open-Loop Traffic}

The first set of simulation experiments studies the performance and the accuracy of the hybrid implementation using open-loop traffic. Identical and independent Exponential On/Off sources, as described in Section 4.3, are used. The sources generate unidirectional traffic to the sinks.

In these experiments, the number $N$ of router hops in the network is varied from 1 to 16 . The number of foreground flows $K$ is varied from 1 to 4 , while the number of background flows $L$ is varied from 2 to 32 . Each simula- 


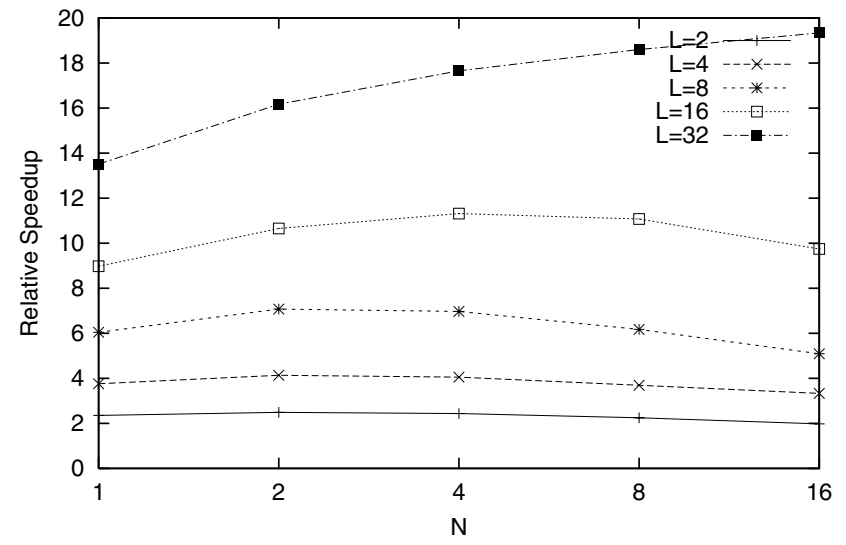

(a) Medium load, $\mathrm{K}=1$

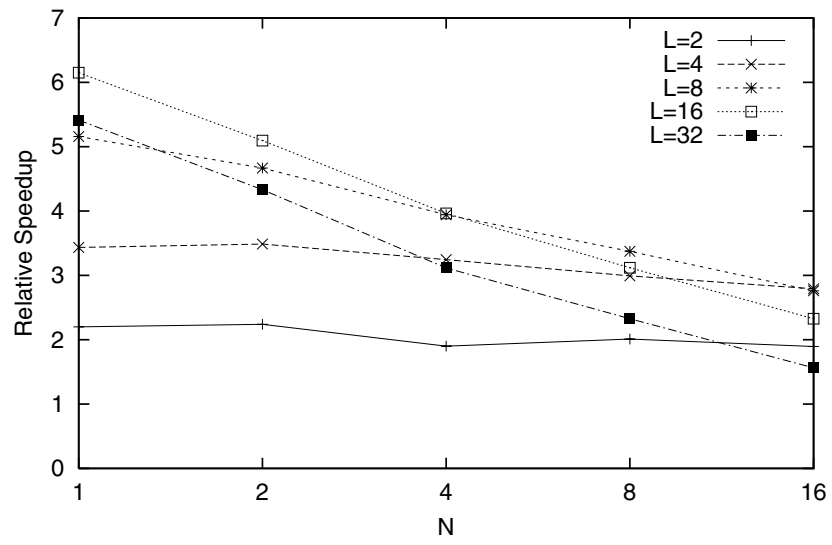

(b) Heavy load, $\mathrm{K}=1$

Figure 5. Plots of relative speedup of hybrid implementation vs $\mathbf{N}$ for (a) Medium load with $\mathrm{K}=1$ and (b) Heavy load with $\mathrm{K}=1$.

tion configuration was run 10 times using different random number seeds, with the average values for the performance metrics calculated from the 10 runs. Each run simulated 600 seconds.

Three different levels of network load are studied: Light, Medium, and Heavy. These scenarios correspond to no packet loss, low packet loss, and high packet loss, respectively. For Light load, the backbone link capacities are set to $C=M * 10 \mathrm{Mbps}$, which is double the capacity required to handle $M$ flows that are simultaneously in the On state. The average offered network load is $25 \%$, and queuing within the network is negligible. (For space reasons, these results are omitted from the paper.) For Medium load, the backbone link capacities are set to $C=M * 3.5 \mathrm{Mbps}$. The average offered network load is about $70 \%$, though the peak load when many flows are active can clearly exceed the network capacity. These transient overloads induce queuing delays at the points of congestion, and occasional losses of packets. For Heavy load, the backbone link capacities are set to $C=M * 2.5 \mathrm{Mbps}$. This is exactly enough capacity to handle the long-term average load from $M$ On/Off sources. Significant queuing delays and packet losses can occur when instantaneous demand exceeds this capacity.

\subsection{Simulation Performance}

Figure 5 shows the relative speedup results for the hybrid simulation compared to the packet simulation, for a single foreground flow in the network $(K=1)$. Figure 5(a) presents the results for Medium load, while Figure 5(b) presents the results for Heavy load. Each line on the graphs represents a different number of background flows; these are fluid flows in the hybrid simulation, and packet flows in the packet simulation. In both graphs, the relative speedup values are shown as a function of network size $N$, the number of router hops. Note that the horizontal axes use a logarithmic scale.

The simulation results in Figure 5 show (as expected) that the hybrid simulation is faster than the packet simulation. The relative speedup advantage varies from a factor of 2 to a factor of 20, depending on the network topology and traffic model used.

Figure 5(a) presents the results for Medium network load. Here, the performance advantage of the hybrid simulation clearly increases as the number of (fluid) background flows increases. However, there is a "diminishing returns" effect as well, which limits the relative speedup achieved as the network size $N$ increases. This phenomenon is attributed to the "ripple effect": as the number of possible congestion points in the network increases, it is more likely that flow interactions and packet losses trigger rate changes in the fluid flows, increasing the number of simulation events in the hybrid model. The diminishing returns effect is not present for the Light load scenario: relative speedup always improves as $N$ is increased and as $L$ is increased.

The impact of the "ripple effect" is even more pronounced in Figure 5(b), for Heavy network load. The relative speedup values here are much lower than in Figure 5(a). Furthermore, adding more (fluid) background flows does not always improve speedup. Initially, as the number of background flows is doubled from 2 to 4 , and from 4 to 8 , the speedup advantage of the hybrid simulation improves. However, doubling $L$ again to 16 improves speedup only marginally on small networks, and makes speedup worse (compared to $L=8$ ) for large networks. Doubling $L$ again to 32 reduces the speedup advantage (compared to $L=16$ ) 


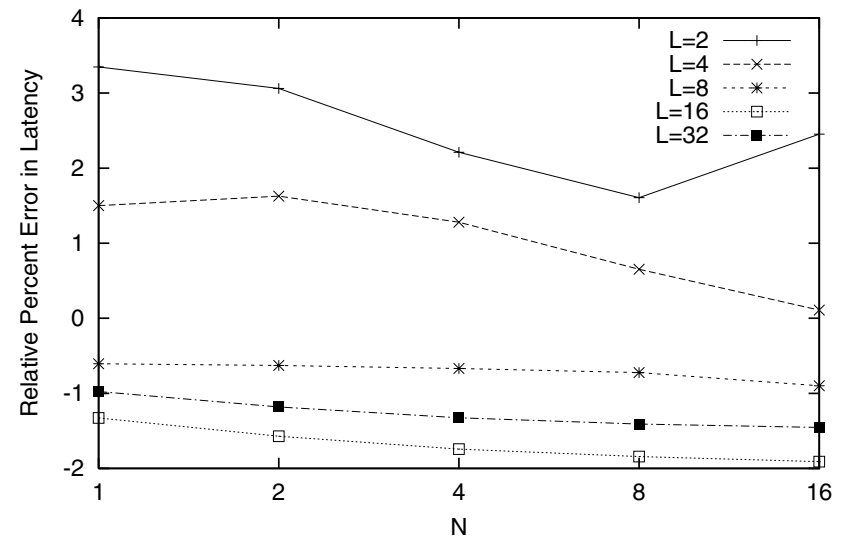

(a) Medium load, $\mathrm{K}=1$

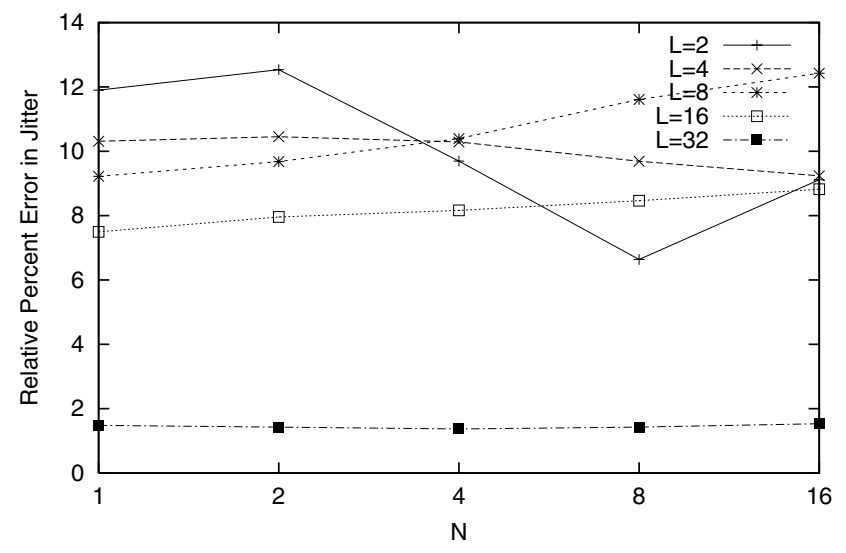

(c) Medium load, $\mathrm{K}=1$

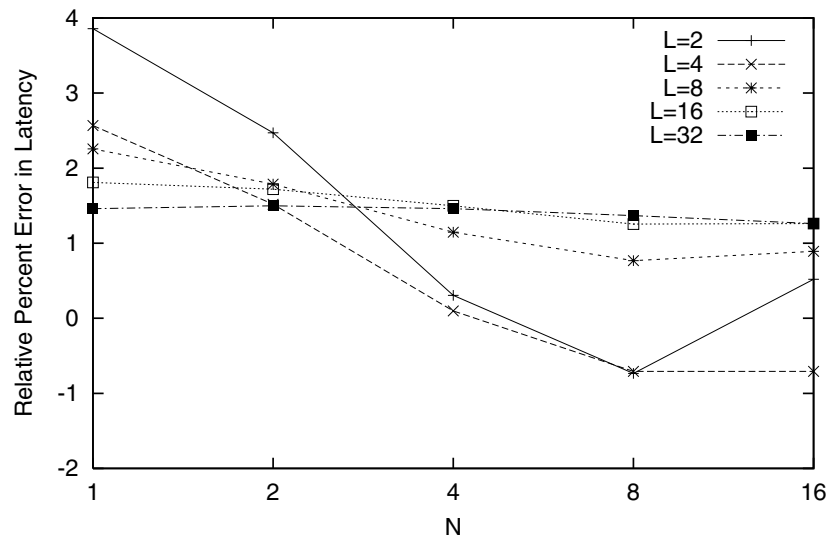

(b) Heavy load, $\mathrm{K}=1$

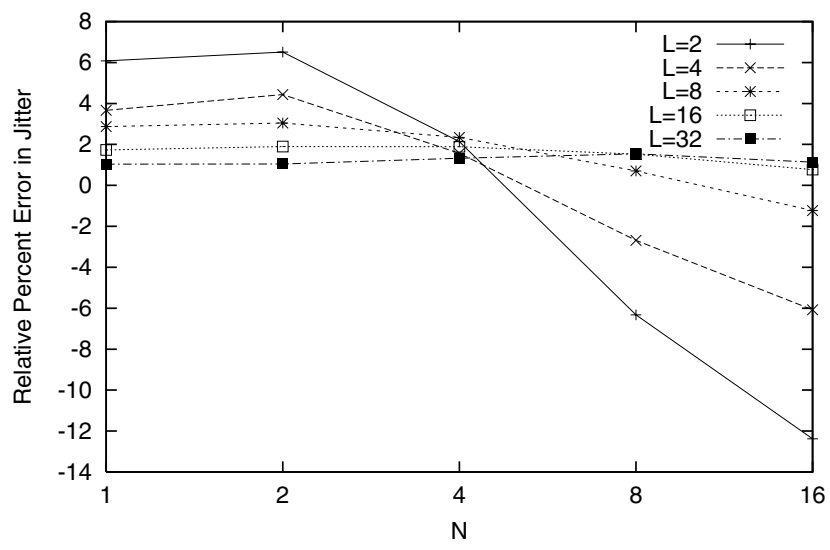

(d) Heavy load, $\mathrm{K}=1$

Figure 6. Plots of relative percent error in latency vs $N$ for (a) Medium load with $K=1$ and (b) Heavy load with $K=1$ and relative percent error in jitter vs $N$ for (c) Medium load with $K=1$ and (d) Heavy load with $K=1$.

of the hybrid simulation across the full range of network sizes considered. In general, speedup tends to decrease as $N$ increases, due to the increased ripple effect. For larger $L$ or larger $N$, the hybrid implementation could be slower than the packet implementation.

The relative speedup for $K=4$ foreground flows at Medium load (not shown here) is about half that in Figure 5(a) for $K=1$. This decrease makes sense, given the increase in the number of packet events in the simulation. The qualitative shape of the speedup curves remains the same as the number of foreground flows is varied.

\subsection{Simulation Accuracy}

Figure 6 shows the simulation accuracy results for the Medium and Heavy network load scenarios, for $K=1$ foreground flow. These graphs present the relative percentage error in mean end-to-end transfer latency (Figures 6(a) and (b)) and jitter (Figures 6(c) and (d)) for the hybrid sim- ulation, compared to the packet simulation.

These results show that the relative error in mean transfer latency is low (e.g., less than $4 \%$ for all cases depicted in Figure 6). Results for Light load (not shown here) also have a relative error less than $4 \%$, as expected.

The relative error in the jitter metric tends to be higher, though it is still under 15\% in all cases considered. This observation implies that the distribution of end-to-end delays is similar in both the packet and hybrid simulations. For Light load (not shown here), relative error in jitter is almost $-100 \%$. This is because there is little or no jitter in the hybrid simulation due to negligible queueing, whereas there is some jitter in the packet simulation due to some queueing.

The results in Figure 6 also show that the relative error in latency and jitter tends to decrease (and stabilize) as the number of background flows is increased. One possible explanation is that as the number of background flows increases, the flow interactions at the buffers increase, result- 


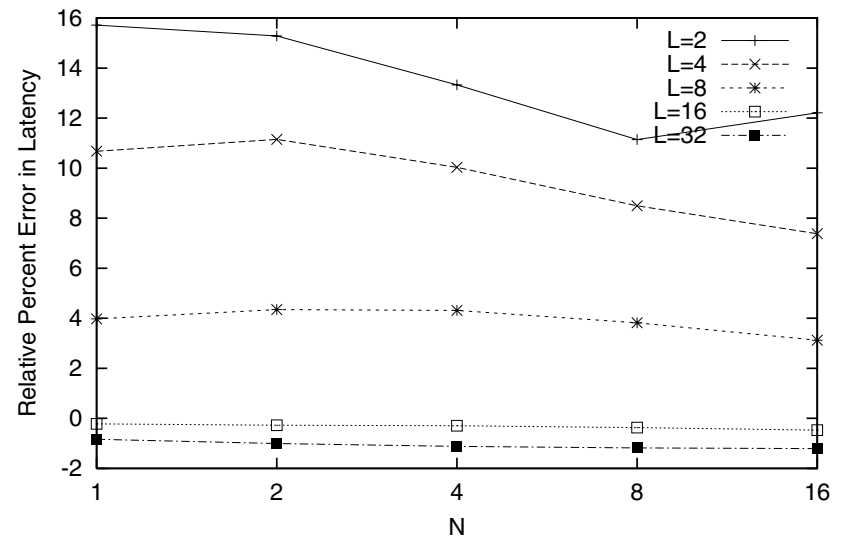

(a) Medium load, $\mathrm{K}=4$

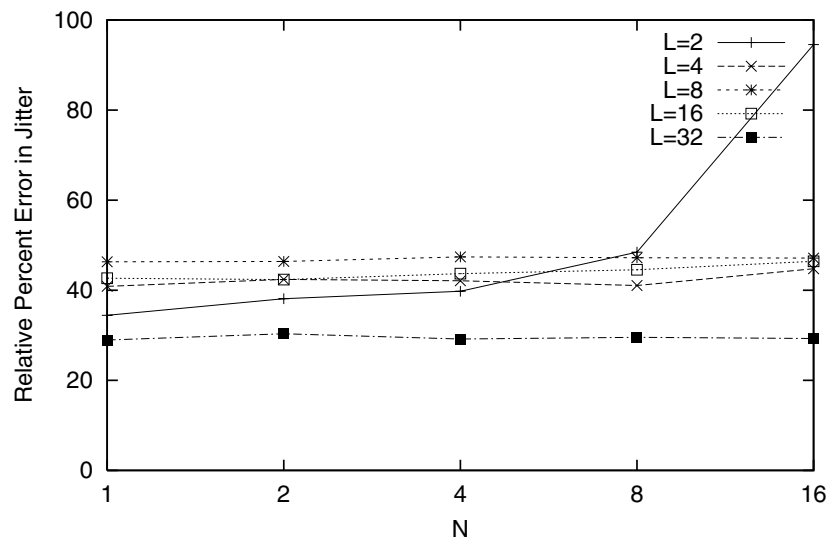

(b) Medium load, $\mathrm{K}=4$

Figure 7. Plots of (a) relative percent error in latency vs $\mathrm{N}$ for Medium load with $\mathrm{K}=4$ and (b) relative percent error in jitter vs $\mathbf{N}$ for Medium load with $\mathrm{K}=4$.

ing in fluid dynamics that better approximate the statistical multiplexing in the packet simulation. Furthermore, the variance of the background traffic tends to decrease relative to the mean as sources are aggregated, since the sources are independent.

Increasing the number of foreground flows tends to increase the relative error in both the latency and jitter metrics. This effect is illustrated in Figure 7 for $K=4$. This effect is attributed to the dynamics of the packet rate estimation algorithm.

\section{Results for Closed-Loop Traffic}

The second set of simulation experiments studies the accuracy of the hybrid simulation for closed-loop traffic. A Web client/server model is used to model a single foreground flow, with multiple TCP transfers taking place (one at a time, 100 seconds apart) on this foreground flow during the simulation. Background flows use the Exponential On/Off source model (packet or fluid). The unidirectional background traffic competes with the TCP data packets flowing from the server to the client. TCP acknowledgment packets return on the uncontested reverse channel.

The purpose of the experiment is to compare TCP transfer durations for both the packet and hybrid simulations. In particular, we study the cumulative effect of the relative errors in packet transfer latencies on the overall TCP transfer duration observed by a Web client.

For this experiment, we consider TCP transfer sizes ranging from $1 \mathrm{~KB}$ to $50 \mathrm{~KB}$, which spans the typical range of Web document sizes. We focus only on the simulation accuracy results for this performance metric, using a single simulation run. The speedup results are not presented, since they are overly optimistic: they are dominated by efficient fluid-only execution of the background flows in between the arrivals of the foreground TCP transfers.

\subsection{Simulation Accuracy}

Figure 8 presents the simulation results from these experiments. The first row of graphs (Figures $8(a)$ and (b)) is for $L=8$ background flows at $70 \%$ network load, while the second row of graphs (Figures 8(c) and (d)) shows the results for $L=32$ background flows at $90 \%$ network load. The load values represent the average offered load from the background flows, since the foreground flow is inactive most of the time. In all cases, there is only a single foreground TCP flow. The first column of graphs (Figures 8(a) and (c)) is for a single hop network $(N=1)$, while the second column of graphs (Figures 8(b) and (d)) is for $N=8$. The packet loss ratios indicated are for the background flow that traverses the entire network; the foreground flow should experience a similar packet loss ratio. In general, the average packet loss ratio increases with the number of hops traversed.

These four graphs use scatterplots to present the simulation results. Each point in the plots represents the TCP transfer duration (in seconds, on the vertical axis) for a completed TCP connection with the transfer size in packets indicated on the horizontal axis. (Note that the vertical axis is log scale, while the horizontal axis is linear scale.) Each '+' represents a transfer time result from the packet simulation, while each ' $x$ ' represents a result from the corresponding hybrid simulation.

The results in Figure 8 show that there is close agreement between the TCP transfer durations reported by the packet and hybrid simulations. For many transfer sizes, the '+' and 'x' points coincide, indicating that the hybrid model 


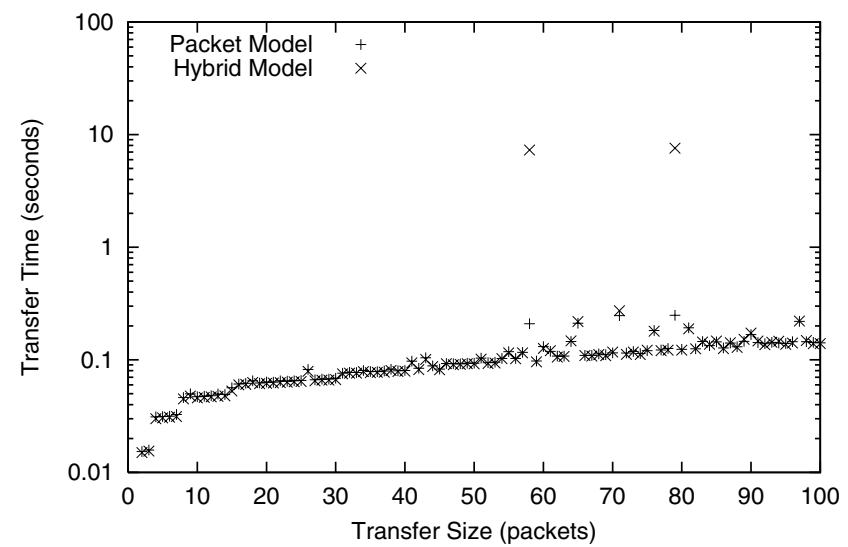

(a) $\mathrm{N}=1, \mathrm{~L}=8$, packet loss $=0.1 \%$

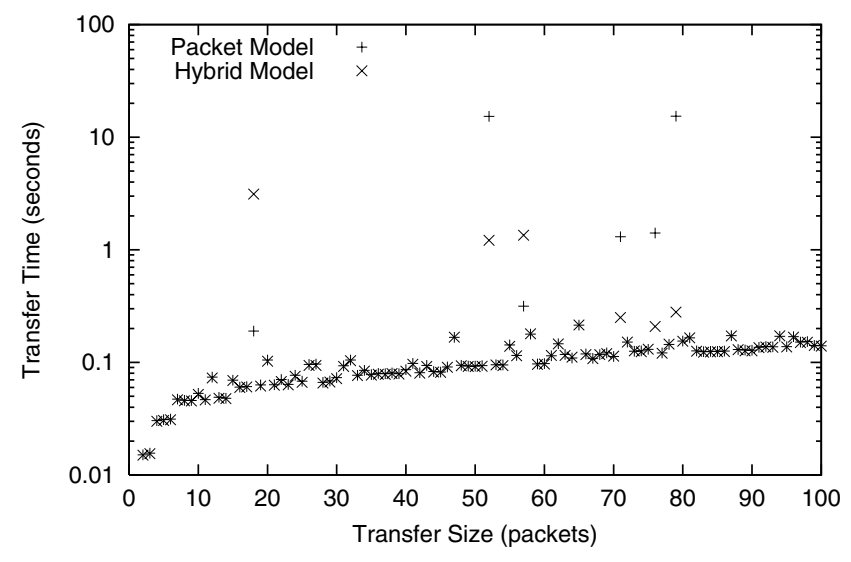

(c) $\mathrm{N}=1, \mathrm{~L}=32$, packet loss $=0.5 \%$

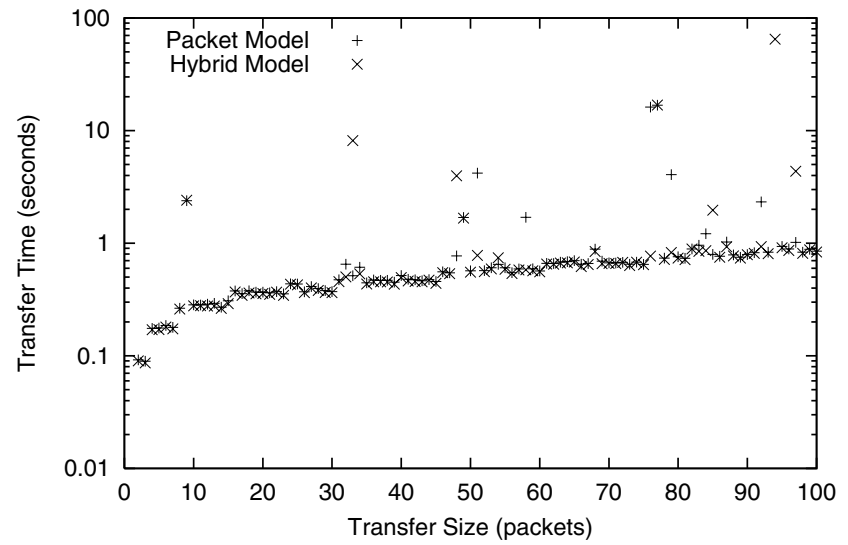

(b) $\mathrm{N}=8, \mathrm{~L}=8$, packet loss $=1 \%$

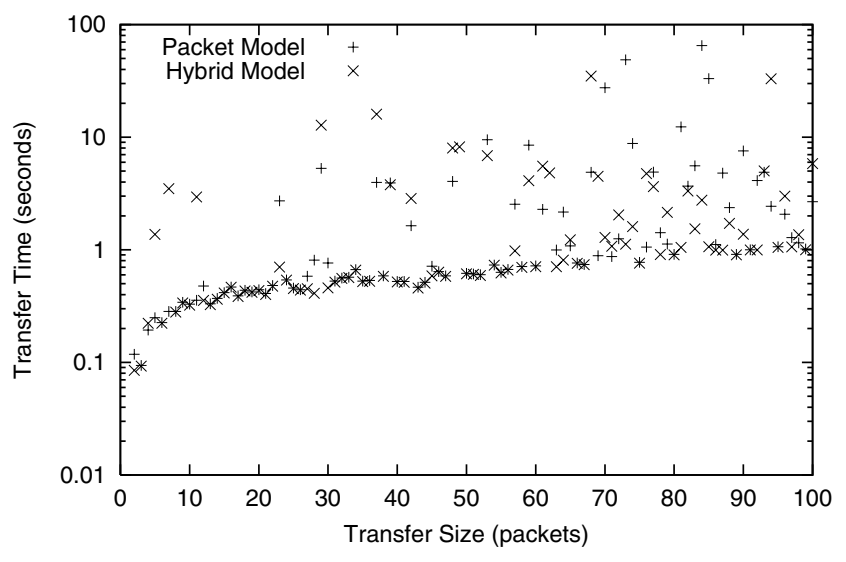

(d) $\mathrm{N}=8, \mathrm{~L}=32$, packet loss $=3 \%$

Figure 8. Plots of transfer time vs transfer size for (a) $N=1, L=8$, packet loss $=0.1 \%$ (b) $N=8, L=8$, packet loss $=1 \%$ (c) $\mathrm{N}=1, \mathrm{~L}=32$, packet loss $=0.5 \%$ and (d) $\mathrm{N}=8, \mathrm{~L}=32$, packet loss $=3 \%$.

provides an excellent approximation of the TCP transfer duration in the packet simulation.

All four graphs show a distinctive structure representative of TCP. In particular, as the transfer size is increased, a step-like structure appears, indicating the additional roundtrip times required to complete the transfer. The step-like structure is most evident at Light load (not shown here), since there is little or no queuing delay in the network. At higher loads, queuing delays, packet losses, and retransmissions can add to the transfer duration, producing points above the lower bound corresponding to network round-trip times.

The encouraging observation is the close agreement in transfer durations even for some points above the TCP lower bound. This suggests that the hybrid model produces queuing delays and packet losses that are similar to the packet model, triggering similar TCP behaviors at the endpoints. In addition, these results suggest that relative errors in modeling end-to-end packet transfer delay do not accumulate; rather, they seem to average out over a multipacket transfer. This observation is particularly promising for network emulation purposes.

The fidelity of the hybrid simulation is better for the single-hop network (Figures 8(a) and (c)) than for $N=8$ (Figures 8(b) and (d)), and better at 70\% load (Figures 8(a) and (b)) than at $90 \%$ load (Figures 8(c) and (d)). There are some discrepancies between ' + ' and ' $x$ ' points in all four graphs. These discrepancies may represent packet loss events triggered in one simulation model but not the other, or simply packet losses that occur at different places within the multi-packet transfer. Nevertheless, the distribution of transfer durations appears similar in both the packet and hybrid models.

\section{Conclusions and Future Work}

This paper presented a hybrid network simulation model that integrates both packet and fluid flows. Initial results 
show up to 20 times speedup using the hybrid approach over using a purely packet-based approach. Accuracy is within $4 \%$ for latency and $15 \%$ for jitter in many cases, though accuracy decreases as the number of packet foreground flows increases. Performance improves when more fluid background flows are modeled, as long as congestion is not too high. Performance decreases in cases where a large congested network leads to the ripple effect dominating. Increasing the ratio of packet flows to fluid flows also decreases performance.

In the network model for which simulation results are presented, each link is modeled to have approximately the same congestion level. Therefore, each backbone link was a bottleneck when congestion levels were high. Since it is unusual for all links to be bottlenecks, performance levels achieved using these techniques with real simulation models is likely to be much higher than for the benchmark results presented. However, this approach may not be appropriate for use in models with large numbers of packet flows and high congestion.

Further research is required to fully understand the impact the hybrid implementation has on accuracy and performance. It is possible that components of the hybrid implementation could be improved to offer greater accuracy and performance. Also, studies need to be performed to determine the performance capabilities using this approach along with parallel discrete event simulation techniques. Finally, experiments need to be performed to determine how much performance can be increased for real-time network emulation experiments using this approach.

\section{Acknowledgments}

Financial support for this research was provided by NSERC (Natural Sciences and Engineering Research Council of Canada), iCORE (Informatics Circle of Research Excellence) and ASRA (Alberta Science and Research Authority). Other members of the IP-TN development team include Mike Bonham, Roger Curry, Mark Fox, Hala Taleb, Kitty Wong and Xiao Zhong-e. The authors wish to thank Christiane Lemieux, who supervised a course project conducted by Cameron Kiddle on fluid modeling techniques. Also, the authors wish to thank the anonymous referees for their constructive comments regarding the paper.

\section{References}

[1] R. M. Fujimoto, T. McLean, K. Permualla, and I. Tacic. Design of high performance RTI software. In Proceedings of the Fourth IEEE Workshop on Distributed Simulation and Real-Time Applications, pages 89-96, 2000.

[2] G. Kesidis, A. Singh, D. Cheung, and W. W. Kwok. Feasibility of fluid event-driven simulation for ATM networks. In Proceedings of the IEEE Global Telecommunications Conference, pages 2013-2017, 1996.
[3] B. Liu, D. R. Figueiredo, Y. Guo, J. Kurose, and D. Towsley. A study of networks simulation efficiency: Fluid simulation vs. packet-level simulation. In Proceedings of the Twentieth Annual Joint Conference of the IEEE Computer and Communications Societies (INFOCOM), pages 1244-1253, 2001.

[4] B. Liu, Y. Guo, J. Kurose, D. Towsley, and W. Gong. Fluid simulation of large scale networks: Issues and tradeoffs. In Proceedings of the International Conference on Parallel and Distributed Processing Techniques and Applications, pages 2136-2142, 1999.

[5] B. Melamed, S. Pan, and Y. Wardi. Hybrid discretecontinuous fluid-flow simulation. In Scalability and Traffic Control in IP Networks, Sonia Fahmy, Kihong Park, Editors, Proceedings of SPIE Vol. 4526, pages 263-270, 2001.

[6] V. Misra, W. Gong, and D. Towsley. Fluid-based analysis of a network of AQM routers supporting TCP flows with an application to RED. Computer Communication Review, 30(4):151-160, 2000.

[7] D. Nicol. Discrete event fluid modeling of TCP. In Proceedings of the 2001 Winter Simulation Conference, pages 1291-1299, 2001.

[8] D. Nicol, M. Goldsby, and M. Johnson. Fluid-based simulation of communication networks using SSF. In Proceedings of the 11th European Simulation Symposium, pages 270274, 1999.

[9] J. M. Pitts. Cell-rate modelling for accelerated simulation of ATM at the burst level. IEE Proceedings-Communications, 142(6):379-385, 1995.

[10] G. F. Riley, R. M. Fujimoto, and M. H. Ammar. A generic framework for parallelization of network simulations. In Proceedings of the Seventh International Symposium on Modeling, Analysis and Simulation of Computer and Telecommunication Systems, pages 128-135, 1999.

[11] G. F. Riley, T. M. Jaafar, and R. M. Fujimoto. Integrated fluid and packet network simulations. In Proceedings of the Tenth IEEE International Symposium on Modeling, Analysis and Simulation of Computer and Telecommunication Systems, pages 511-518, 2002.

[12] R. Simmonds, R. Bradford, and B. Unger. Applying parallel discrete event simulation to network emulation. In Proceedings of the 14th Workshop on Parallel and Distributed Simulation, pages 15-22, 2000.

[13] T. K. Yung, J. Martin, M. Takai, and R. Bagrodia. Integration of fluid-based analytical model with packet-level simulation for analysis of computer networks. In Internet Performance and Control of Network Systems II, Robert D. van der Mei, Frank Huebner-Szabo de Bucs, Editors, Proceedings of SPIE Vol. 4523, pages 130-143, 2001.

[14] X. Zeng, R. Bagrodia, and M. Gerla. GloMoSim: A library for parallel simulation of large-scale wireless networks. In Proceedings of the 12th Workshop on Parallel and Distributed Simulation, pages 154-161, 1998. 